\title{
The variable sources responsible for the photometric behaviour of OJ 287 in the IR-optical-UV region during 1993-1996
} \author{
J.N. Gonzalez-Perez ${ }^{4}$ \\ 1 Astronomical Institute of St. Petersburg State University, 198904 St. Petersburg, Russia \\ 2 Tuorla Observatory, Tuorla 21500, Piikkiö, Finland \\ 3 Boltwood Observatory, 1655 Main St., Stittsville, Ontario, Canada \\ ${ }^{4}$ Instituto de Astrofisica de Canarias, La Laguna, 38200 Tenerife, Spain
}

V.A. Hagen-Thorn ${ }^{1}$, S.G. Marchenko ${ }^{1}$, L.O. Takalo ${ }^{2}$, A. Sillanpää ${ }^{2}$, T. Pursimo ${ }^{2}$, P. Boltwood ${ }^{3}$, M. Kidger ${ }^{4}$, and

Received October 20, 1997; accepted June 22, 1998

\begin{abstract}
An analysis is performed of multicolour observations of OJ 287 carried out during the OJ-94 Project. It is found that in the optical region the variable sources have the same power-law spectrum $\left(F_{\nu} \sim \nu^{-1.5}\right)$ irrespective of flux level. In the IR the spectrum flattens during the flare possibly because of synchrotron self-absorption. The comparison of the outburst of 1994 with those of 1971 and 1983 shows that there is a clear correlation between the power of outburst and colour indices of the variable component in each event: the larger is the outburst, the bluer is the variable source, i.e. in more powerful outbursts the energy spectrum of relativistic electrons is flatter.
\end{abstract}

Key words: BL Lac objects; OJ 287 — infrared: galaxies

\section{Introduction}

OJ 287 is one of the best studied blazars (e.g. Takalo 1994). After constructing its historical light curve Sillanpää et al. (1988) found a periodicity in the strong optical outbursts of the object (with $P \approx 11.6$ years) and predicted that the next large flare should occur in 1994. This prediction was based on a binary black hole model for OJ 287 (see also Lehto \& Valtonen 1996). Verification of this prediction is one of the goals of the international OJ-94 project, during which the object has been intensively monitored at all available wavelengths since fall 1993 (Kidger et al. 1998). In November 1994 the brightness increase in the IR-optical and UV regions was observed, confirming the prediction (Sillanpää et al. 1996a). This is the first case when a predicted photometric behaviour of extragalactic object has been confirmed. Another large and expected flare was observed during December 1995 (Sillanpää et al. 1996b).
By current understanding of the blazar emission the most of it at lower frequencies (in radio bands perhaps all) is due to synchrotron radiation coming from the jet (e.g. Urry \& Padovani 1996). The synchrotron nature of the emission is supported by the observed power-law type spectra and the strong and variable polarization (e.g. Efimov \& Shakhovskoy 1996).

This paper is devoted to the analysis of the OJ 287 colour behaviour in IR-optical-UV region before and during the outburst.

\section{Observational data and their processing}

The OJ-94 Project has collected a large data base on OJ 287 (Pursimo \& Lehto 1996; Sillanpää et al. 1996a,b; Kidger et al. 1998) with good time coverage, especially in the optical region. This is by far the most complete light curve ever obtained for an extragalactic object, with over 3000 data points in the $V$-band and slightly less in the other optical bands (Table 1). All the collected data is archived in Tuorla Observatory (Pursimo \& Lehto 1996) and can be obtained from the Project WEB site: http://astro.utu.fi/oj94/. In UV and IR the data are somewhat sparse but the IR light curves are more detailed than what has previously been observed.

UV-observations were carried out with IUE in Nov.Dec. 1994, during the optical flare, in two spectral bands (for details see Pian et al. 1996). Seven flux measurements are available both at $\lambda 2550$ and at $\lambda 1550$.

The majority of the IR data were obtained during 1993/1994 observational season with two 1.5-m telescopes; TIRGO at Gornergrad, Switzerland and TCS on Teide Observatory, Tenerife, Spain (Kidger \& Gonzalez-Perez 1994). The light curves are quite well covered, especially from early December 1993 till April 1994. A few additional observations were obtained in autumn 1994. 
Table 1. Basic information about optical observations

\begin{tabular}{ccc}
\hline $\begin{array}{c}\text { Photometric } \\
\text { band }\end{array}$ & $\begin{array}{c}\text { The total amount } \\
\text { of photometric } \\
\text { estimations }\end{array}$ & $\begin{array}{c}\text { The number } \\
\text { of telescopes } \\
\text { (observatories) }\end{array}$ \\
\hline$U$ & $69(1)$ & 4 \\
$B$ & $335(10)$ & 10 \\
$V$ & $3179(67)$ & 15 \\
$R$ & $3465(88)$ & 13 \\
$I$ & $2302(57)$ & 9 \\
\hline
\end{tabular}

The data base contains 75 points in the $K$ band, 73 in $H$ and 47 in $J$.

The optical observations discussed here cover the time range from September 1993 till March 1996. Table 1 contains the basic information about optical observations: photometric band, the amount of individual brightness measurements and the number of telescopes (or observatories) participating in observations (see also Sillanpää et al. 1996a,b; Kidger et al. 1998). The majority of $V, R, I$ data have been obtained by Boltwood.

An examination of the whole data base shows that there are some observations with large errors. In the archive we have saved ALL the data sent by the different observers without special quality check. Bearing in mind that it is always better to reject shady data than to use them in the analysis we have decided to remove such measurements from the data base. To find an acceptable level of observational errors we used the following method. In each spectral band the magnitudes were transformed to fluxes using absolute calibration by Mead et al. (1990). Then the variability ranges were divided in segments of $1 \mathrm{mJy}$ length. The mean flux value $\bar{F}_{j}$, corresponding mean observational error $\bar{\sigma}_{j}$ and its dispersion $\Delta_{j}^{2}=\frac{\sum_{k=1}^{n_{j}}\left(\sigma_{k}-\overline{\sigma_{j}}\right)^{2}}{n_{j}-1}$ were calculated for each segment $j$ which contains $n_{j}$ points. An observation was considered as unreliable when its relative error $\sigma_{k} / F_{k}$ exceeds $\left(\bar{\sigma}_{j}+3 \Delta_{j}\right) / \bar{F}_{j}$.

Figure 1 demonstrates the method of data reduction for the $V$ band. Crosses give relative errors for all individual observations. Solid line is the exponential approximation of the dependence on $F$ of the values $\left(\bar{\sigma}_{j}+3 \Delta_{j}\right) / \bar{F}_{j}$ (triangles in Fig. 1). All points lying above this line were rejected from further consideration. The number of data points removed at this stage is indicated in the second column of Table 1 in brackets. Note that the number of rejected data points is less than $3 \%$ of the data set.

The next step is to check the data homogeneity. Figure 2 shows a fragment of the light curve in the $V$ band for 1993/94 and 1994/95 observational seasons. The data of various authors are plotted by different symbols. The examination of the figure (and the whole data base also) reveals that the $V$ band light curves of various observers agree quite well with each other (see also Sillanpää et al. 1996a,b).

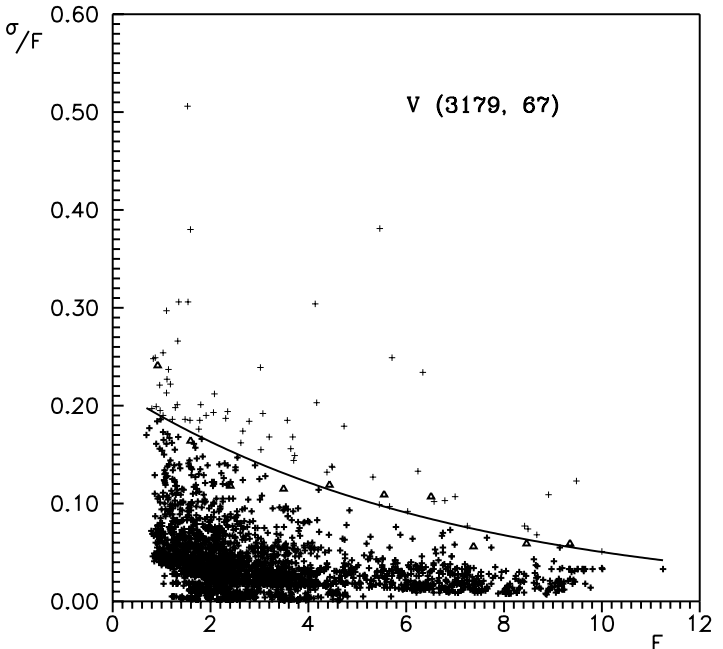

Fig. 1. $V$ band; the dependence of individual observational relative errors on flux (see text)

To examine the agreement in other bands the dependences of colour indices $B-V, V-R$ and $V-I$ on $V$ magnitude were constructed. As an example the dependence of $V-R$ versus $V$ is shown in Fig. 3. One can see that there are some points very much scattered relative to a general dependence. They are labeled by numbers being the last four figures of JD. These points as well as analogous points on the other graphs were also rejected from data base as unreliable.

At the same time all three graphs show that systematical shifts between the colour indices found by various observers are smaller than $0.1^{\mathrm{m}}$ (if any). Therefore no correction for this effect was made.

After rejecting all shady data the mean values of fluxes for each JD were calculated taking into account the errors of individual observations. Note however, that here we have ignored the microvariability observed during some nights (e.g. Dultzin-Hacyan et al. 1997). Values for $F$ and their mean square errors were found for 36 dates in $U$ band, 175 in $B, 458$ in $V, 406$ in $R, 321$ in $I, 28$ in $J, 36$ in $H$ and 37 in $K$ band.

All the data (including those for UV bands) were corrected for galactic absorption using the standard curve of interstellar reddening and $A_{V}=0.06^{\mathrm{m}}$ according to Smith et al. (1987). This data base was used for further analysis.

\section{The method of the analysis of multicolour photometric data}

For the analysis of colour behaviour we use the method first proposed by Choloniewski (1981) and considered recently in detail by Hagen-Thorn (1997). This method has been used by us several times, in particular, to study the previous outbursts of OJ 287 (Hagen-Thorn 1987; HagenThorn et al. 1991, 1994). 


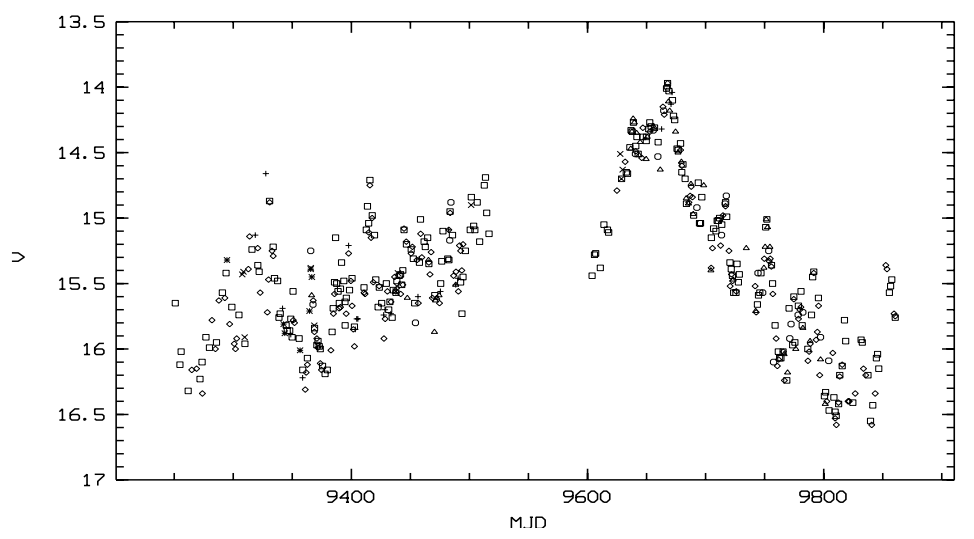

Fig. 2. The $V$ band light curve of OJ 287 for seasons 1993/94 and 1994/95. The different symbols correspond to different observers: $\square$-Boltwood, $\triangle$ - RoboScope, $\diamond$ - Italian, $\circ$ - Crimean Observatory, + - IAC-80 telescope, $\times$ - Nordic Optical Telescope, * - Jakobus Kapteyn Telescope

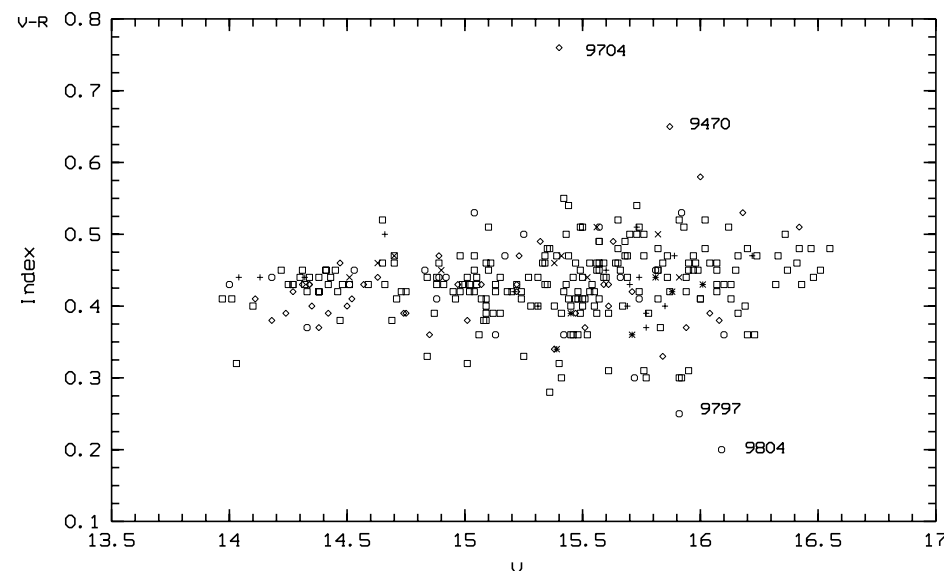

Fig. 3. The dependence of the colour index $V-R$ on the $V$ magnitude (the symbols are the same as in Fig. 2)

In the frame of a two-component model (constant component + variable source) the method allows to obtain the relative spectral energy distribution of the source responsible for variability if the source has variable flux but unchanged spectral shape. If this is the case, the points corresponding to the observed fluxes at different times should lie on a straight line in the $n$-dimensional flux space $\left\{F_{1}, \ldots F_{n}\right\}$ (where $n$ is the number of photometric bands) and consequently on straight lines in the planes $\left\{F_{i}, F_{j}\right\}$. Their slopes are the flux ratios of the variable component in the bands under consideration. Plotting these ratios as a function of frequency gives the spectral energy distribution of the variable component in relative units.

Thus if observational points in fact lie on the straight lines within observational errors one can conclude that the model of single variable source with unchanged spectral energy distribution is valid. The slopes of the lines (fitted by the method of orthogonal regression (Wald 1940) used in the case of accidental errors existence in both axes) give above mentioned ratios (the relative spectral energy distribution).

\section{Mean observed spectrum of the variable component for 1993-1996}

When finding the relative spectral energy distribution we choose the $V$ band as the basic one since there is a maximal data completeness in it (the $V$ band light curve transformed to fluxes is shown in Fig. 4).

Figure 5 demonstrates the comparison of the fluxes in the optical region for the whole data base. It is evident that all points at corresponding graphs lie on straight lines quite well. This is confirmed by the correlation coefficients $r_{\text {iv }}$ (Col. 3 in Table 2) which are very close to 1 in all four cases. (The first two columns of the table contain the band name and corresponding value of $\log \nu$.) The slopes of the lines give the ratios of energy radiated by variable source in $U, B, R$ and $I$ bands to that of $V$ band.

Figure 6 represents the dependences of fluxes at the $\lambda 1550$ (triangles) and $\lambda 2550$ (open circles) on the flux in the $V$ band. They follow the straight lines sufficiently well. These graphs allows us to widen the relative spectral energy distribution to UV.

The comparison of IR fluxes with the flux in the $V$ band is shown in Figs. 7a-c. Here the scatter is much larger 


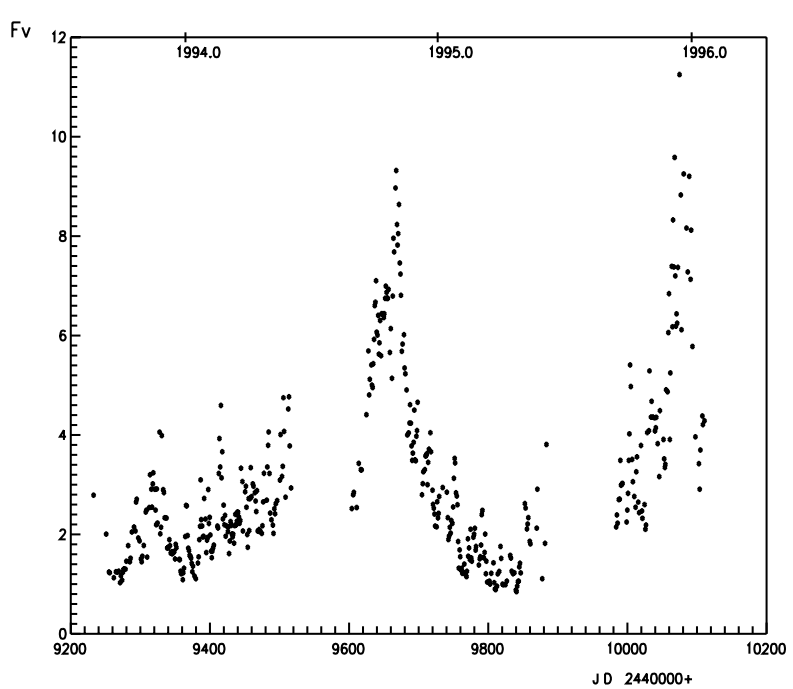

Fig. 4. $V$ band light curve transformed to fluxes

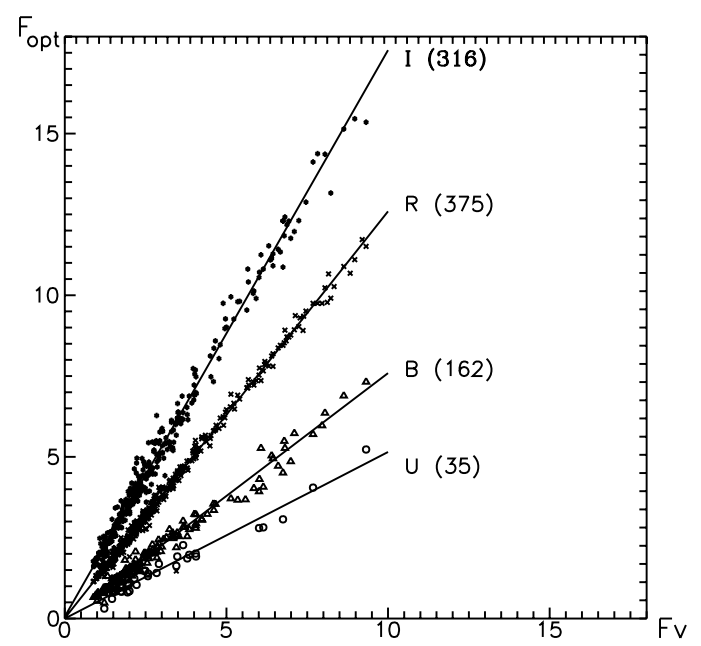

Fig. 5. Comparison of the fluxes in the optical bands

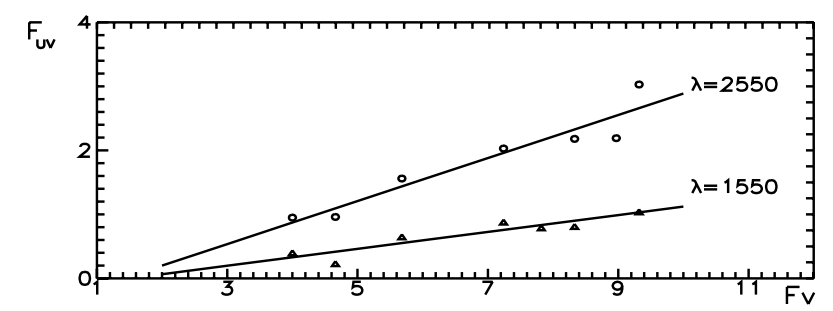

Fig. 6. Comparison of the fluxes in the UV region with that in the $V$ band

than in Figs. 5, 6. Nevertheless in all cases one can try to represent the dependence by single straight line (solid lines in Figs. 7a-c).

The slopes of the lines in Figs. 5-7 give the mean relative spectral energy distribution of the variable component (assumed to be unchanged) in the whole range from $\lambda 1550$ to $K$ found in the two-component model. This distribution is given in the fourth column of Table 2 and in logarithmic
Table 2. The relative spectral energy distribution of variable source (the whole time range)

\begin{tabular}{ccccc}
\hline $\begin{array}{c}\text { Photom. } \\
\text { band }\end{array}$ & $\log \nu$ & $r_{\mathrm{iv}}$ & $\left(F_{\mathrm{i}} / F_{\mathrm{v}}\right)_{\mathrm{var}}$ & $\log \left(F_{\mathrm{i}} / F_{\mathrm{v}}\right)_{\mathrm{var}}$ \\
\hline$K$ & 14.133 & 0.950 & $5.09 \pm 0.26$ & $0.71 \pm 0.02$ \\
$H$ & 14.274 & 0.930 & $3.62 \pm 0.27$ & $0.56 \pm 0.03$ \\
$J$ & 14.380 & 0.949 & $2.80 \pm 0.21$ & $0.45 \pm 0.03$ \\
$I$ & 14.585 & 0.993 & $1.72 \pm 0.01$ & $0.24 \pm 0.01$ \\
$R$ & 14.672 & 0.996 & $1.26 \pm 0.01$ & $0.10 \pm 0.01$ \\
$V$ & 14.737 & 1.000 & 1.00 & 0.00 \\
$B$ & 14.834 & 0.990 & $0.76 \pm 0.01$ & $-0.12 \pm 0.05$ \\
$U$ & 14.915 & 0.982 & $0.52 \pm 0.02$ & $-0.28 \pm 0.02$ \\
$\lambda 2550$ & 15.080 & 0.950 & $0.34 \pm 0.05$ & $-0.47 \pm 0.06$ \\
$\lambda 1550$ & 15.300 & 0.950 & $0.13 \pm 0.03$ & $-0.88 \pm 0.08$ \\
\hline
\end{tabular}

scale in the fifth one. The data of latter column are shown in Fig. 8 (open circles with error bars).

In optical-UV region the relative spectral energy distribution is well represented by the straight line with the slope which gives the spectral index $\alpha=-1.54 \pm 0.05$. One can see however that IR points lie below this line.

\section{Spectra of variable sources found for different observational seasons taken separately}

Careful examination of Figs. 7a-c shows, however, that the representation of the dependences by a single straight lines is a strong simplification. In fact, the highest points are systematically shifted to the right from the straight lines fitting the lower points (these are shown by dashed lines). This shift mounts to $7 \div 10 \sigma_{V}$. The F-test shows that for every graph there is the significant difference (at the confidence level 0.05) between representation the lower points by the solid and dashed lines (for solid one the dispersion is twice as large).

Because the higher points are from 1994/95 (in the flare) and the lower ones are from 1993/94 one can conclude that the properties of IR variable sources in these two time ranges were different. Therefore it is necessary to repeat the analysis for both time intervals taken separately.

Unfortunately, there are very few IR data for 1994/95. We can use the method only to $K$ band, the value of $F_{K} / F_{V}$ being somewhat insecure because there are only three points. Therefore we don't use this value (presented in Table 3 in brackets) in further calculations but give it only for illustration. The F-test shows that representation of the dependence in Fig. 7c by broken (dashed) line is better than by straight (solid) one at the $5-\%$ confidence level. Thus one can be certain that in the flare the slope is flatter for all IR bands.

To be consistent we must divide also the optical data into different time frames. As one can see in Fig. 4 the 

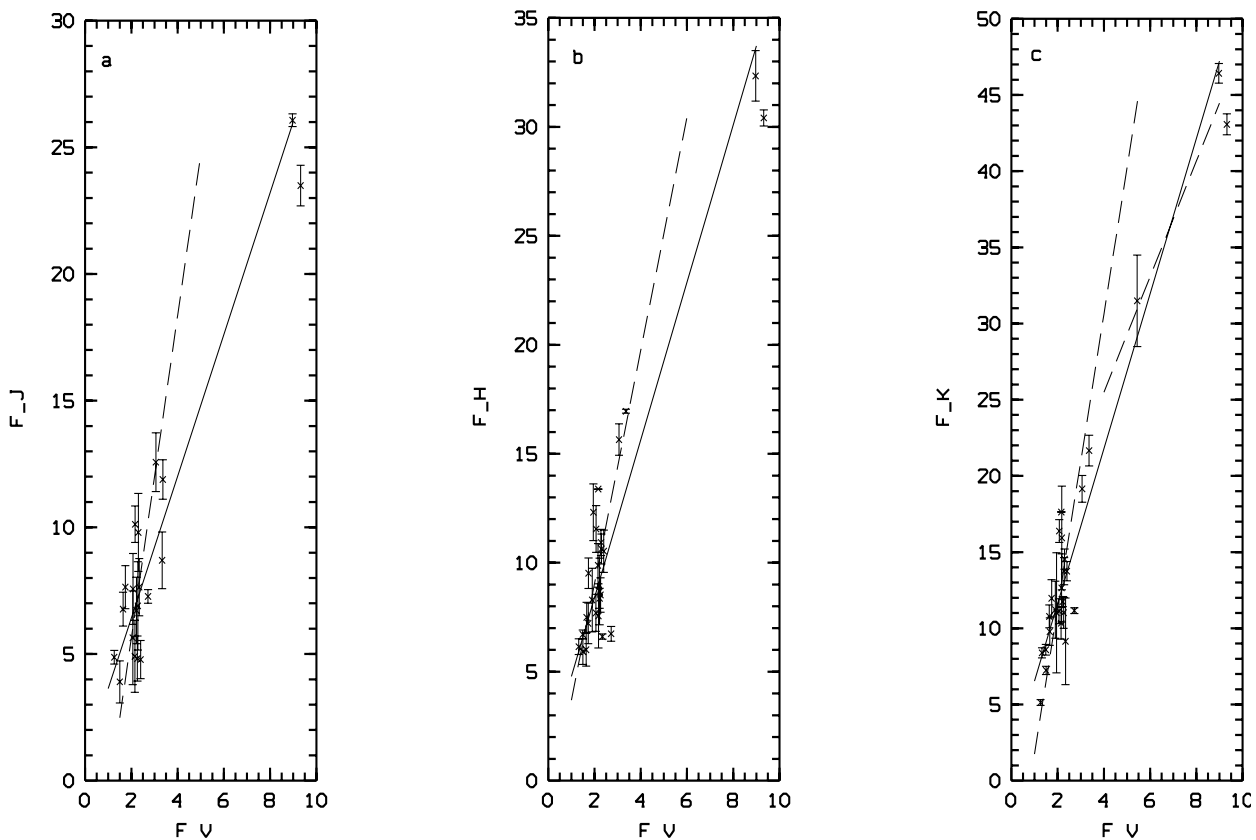

Fig. 7. Comparison of the fluxes in IR and $V$ bands

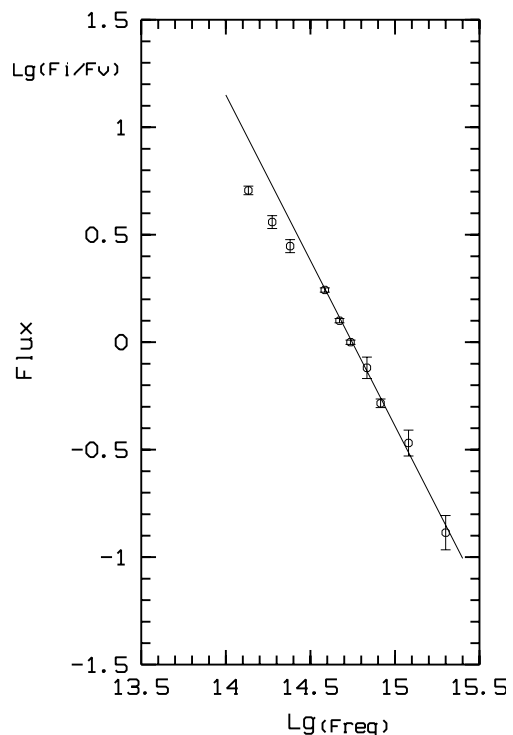

Fig. 8. The mean relative spectral energy distribution of the variable component for 1993-1996; the straight line represents only optical-UV region

light curve has two flares (in 1994/95 and in 1995/96). Therefore we perform the analysis for three time ranges (JD 9250 - 9550, JD 9560 - 9810 and JD $9960-10110$ ).

The results of finding the relative spectral energy distributions of variable components for these three time ranges are given in Table 3 and shown in Fig. 9 by different symbols. Examination of Table 3 and Fig. 9 shows that all three spectra practically coincide with each other in optical region.
The results of fitting the data for first two time intervals in the whole available spectral range are as follows. For 1993/94 season the spectrum may be represented by straight line with the slope $\alpha=-1.61 \pm 0.17$. Evidently the large error is due to $J$-band point which breaches the smoothness of the spectrum expected from physical considerations. If we exclude this point (which has the largest error!) the spectrum from $K$ to $U$ for 1993/94 season is well represented by straight line with the slope $\alpha=-1.55 \pm 0.08$. Unfortunately, in this time range there are no observations of the object in extreme UV.

The spectrum in 1994/95 flare may be also represented from $I$ to $\lambda 1550$ by straight line with the slope $\alpha=-1.52 \pm 0.07$. Unfortunately, in the IR there is only one not too reliable point for $K$ band. But it is evident that it lies below the straight line.

\section{Discussion}

Firstly let us note that both in 1993/94 and 1994/95 the spectra of variable components are well fitted by power law. This result and the existence of high optical polarization (Efimov \& Shakhovskoy 1996) point to their synchrotron nature.

As was mentioned above, in all three seasons the spectral index in optical region was nearly the same irrespective of a flux level. This indicates that the variability wasn't accompanied by changes of energy spectrum of relativistic electrons. Under these circumstances the variability is more probably explained by the variation of the amount of relativistic electrons in the source (HagenThorn et al. 1992). As for flattening the spectrum in the 
Table 3. The relative spectral energy distributions of variable sources for different time ranges

\begin{tabular}{|c|c|c|c|c|c|c|c|c|c|c|}
\hline & $K$ & $H$ & $J$ & $I$ & $R$ & $V$ & $B$ & $U$ & $\lambda 2550$ & $\lambda 1550$ \\
\hline $\log \nu$ & 14.133 & 14.274 & 14.380 & 14.585 & 14.672 & 14.737 & 14.834 & 14.915 & 15.080 & 15.300 \\
\hline \multicolumn{11}{|l|}{ JD 9250-9550 } \\
\hline$r_{\mathrm{iv}}$ & 0.79 & 0.70 & 0.66 & 0.97 & 0.99 & 1.00 & 0.98 & 0.97 & - & - \\
\hline$\left(F_{\mathrm{i}} / F_{\mathrm{v}}\right)_{\mathrm{var}}$ & $9.62 \pm 1.26$ & $5.35 \pm 0.44$ & $6.35 \pm 1.19$ & $1.68 \pm 0.03$ & $1.26 \pm 0.01$ & 1.00 & $0.80 \pm 0.02$ & $0.44 \pm 0.03$ & - & - \\
\hline $\begin{array}{l}\log \left(F_{\mathrm{i}} / F_{\mathrm{v}}\right)_{\text {var }} \\
\text { JD } 9560-9810\end{array}$ & $0.98 \pm 0.06$ & $0.73 \pm 0.03$ & $0.80 \pm 0.08$ & $0.23 \pm 0.01$ & $0.10 \pm 0.01$ & 0.00 & $-0.10 \pm 0.01$ & $-0.35 \pm 0.03$ & - & - \\
\hline$r_{\mathrm{iv}}$ & $(0.96)$ & - & - & 0.995 & 0.998 & 1.00 & 0.988 & 0.985 & 0.950 & 0.950 \\
\hline$\left(F_{\mathrm{i}} / F_{\mathrm{v}}\right)_{\mathrm{var}}$ & $(3.79 \pm 1.11)$ & - & - & $1.73 \pm 0.02$ & $1.25 \pm 0.01$ & 1.00 & $0.76 \pm 0.01$ & $0.53 \pm 0.02$ & $0.34 \pm 0.05$ & $0.13 \pm 0.03$ \\
\hline $\begin{array}{l}\log \left(F_{\mathrm{i}} / F_{\mathrm{v}}\right)_{\text {var }} \\
\text { JD 9960-10110 }\end{array}$ & $(0.58 \pm 0.13)$ & - & - & $0.24 \pm 0.01$ & $0.10 \pm 0.02$ & 0.00 & $-0.12 \pm 0.01$ & $-0.27 \pm 0.02$ & $-0.47 \pm 0.06$ & $-0.88 \pm 0.08$ \\
\hline$r_{\mathrm{iv}}$ & - & - & - & 0.994 & 0.994 & 1.00 & - & - & - & - \\
\hline$\left(F_{\mathrm{i}} / F_{\mathrm{v}}\right)_{\mathrm{var}}$ & - & - & - & $1.66 \pm 0.05$ & $1.24 \pm 0.02$ & 1.00 & - & - & - & - \\
\hline $\log \left(F_{\mathrm{i}} / F_{\mathrm{v}}\right)_{\mathrm{var}}$ & - & - & - & $0.22 \pm 0.02$ & $0.09 \pm 0.01$ & 0.00 & - & - & - & - \\
\hline
\end{tabular}

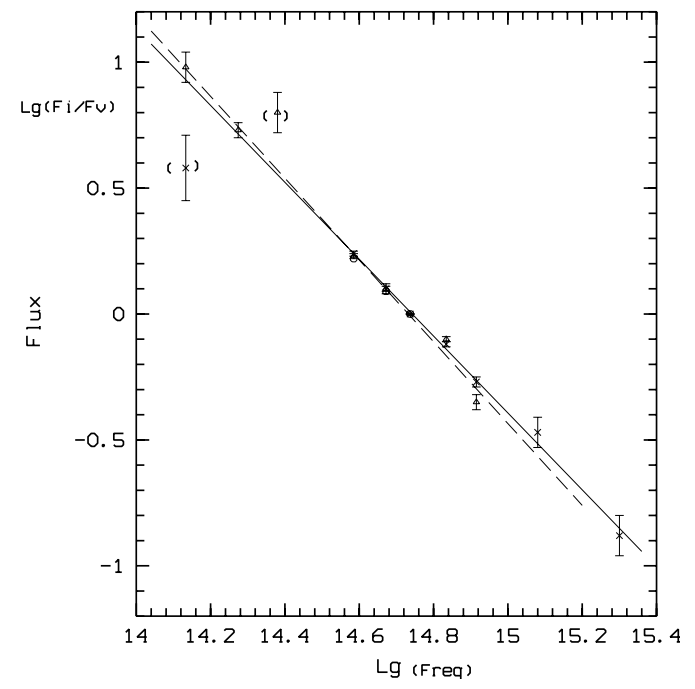

Fig. 9. The relative spectral energy distributions of variable components for three time ranges; 1993/94 - $\triangle$ (dashed line), 1994/95 - ×(solid line), 1995/96 - o

IR over the flare, Hagen-Thorn et al. (1994) supposed that such effect might be due to synchrotron self-absorption. Let us note the lack of 1994 flare at cm-wavelengths certainly due to self- absorption (Marscher 1997).

Up to now the analysis of colour behaviour of OJ 287 was performed for three large outbursts (Hagen-Thorn 1987; Hagen-Thorn et al. 1991, 1994; this paper). These outbursts $(1971,1983,1994)$ are well seen in master light curve of OJ 287 given by Sillanpää et al. (1996a). It is interesting to compare the results for all three outbursts.

For the outburst of 1971 only $U, B, V$ data are available, but the outburst of 1983 was studied in more detail. In all three events the variable source is shown to have variable flux but unchanged spectral energy distribution in optical UV region. For 1971 outburst only the colour indices of variable component $(U-B)_{\text {var }},(B-V)_{\text {var }}$ were found, but in the outbursts of 1983 and 1994 the relative spectral energy distributions in wide spectral range from IR to UV were constructed. In the last two outbursts the relative spectral energy distributions are well represented by the spectrum of synchrotron source; the colour indices of variable source in 1971 outburst also pointed to its synchrotron nature.

The data for all three events are given in Table 4 which contains the maximum brightness in $V$ band in the outburst (Sillanpää et al. 1996a) as well as the colour indices and spectral index in optical region for the variable component.

The examination of Table 4 shows that there is a clear correlation between the maximum brightness in event and colour properties of the variable component. The larger is the outburst the bluer is the variable source. Hence in stronger outburst the energy spectrum of relativistic electrons in the source is flatter.

Let us compare the data for 1983 and 1994 outbursts in more detail. The outbursts show many identical features. In both cases the spectra are rectilinear up to lowest frequencies out of the flare while in the flare they are curved in the IR. Because in the optical region the spectral index within the outburst is the same irrespective of flux level (i.e. the energy distribution of relativistic electrons does not change) one must search for another reason of IR spectrum flattening in the flare. Pacholczyk (1977) points to several such possibilities for synchrotron source. The synchrotron self-absorption is the most alluring because the variability is probably connected with the variations of the amount of relativistic electrons in the source and during the flare the density of electrons in the source should be very high.

But there is a difficulty connected with the fact that usually the flux maximum lies at short centimeter wavelengths. In our case we don't reach the maximum but probably it lies in the region of tens of micrometers. The difference is about 2.5 order of magnitude. Though optical-IR source is much smaller than radio one (as shows the time-scale of variability) and located more close to the jet origin where the magnetic field is higher it is not clear whether this is sufficient to shift the flux maximum to IR wavelengths. May be another reason (e.g. a shift to higher energy of the low-energetic cutoff in energy spectrum of relativistic electrons) should be found.

At the same time there are some differences in spectral energy distribution of synchrotron sources in 1983 
Table 4. Data for three strong outbursts of OJ 287

\begin{tabular}{ccccc}
\hline $\begin{array}{c}\text { Year of } \\
\text { event }\end{array}$ & $\begin{array}{c}\text { for max }) \\
(U-B)_{\text {var }}\end{array}$ & $\begin{array}{c}(B-V)_{\text {var }} \\
\pm \sigma\end{array}$ & $\alpha_{\text {var }}$ \\
$\pm \sigma$ & & \\
\hline 1971 & 12.0 & $-0.72 \pm 0.01$ & $0.33 \pm 0.01$ & - \\
1983 & 12.7 & $-0.62 \pm 0.01$ & $0.44 \pm 0.01$ & -1.3 \\
1994 & 13.8 & $-0.52 \pm 0.01$ & $0.47 \pm 0.01$ & -1.5 \\
\hline
\end{tabular}

and 1994. Besides the difference in spectral slope, the former spectrum clearly demonstrates the high-frequency cutoff (Hagen-Thorn et al. 1994) while the latter one does not. This means that there is a distinction between values of critical frequency depending on maximum energy in the energy distribution of electron and magnetic field strength. Unfortunately, the data don't allow to determine exactly the reason of this distinction.

\section{Conclusions}

The results of multicolour observations of OJ 287 carried out in the frame of OJ-94 Project were analysed by Choloniewski's method with the aim to study the properties of variable sources responsible for photometric behaviour of the object.

It is found that in the optical region the sources have the same power-law spectrum $\left(F_{\nu} \sim \nu^{-1.5}\right)$ irrespective of the flux level (both in and out of flare). This spectrum is of synchrotron nature. In IR the spectrum flattens during the flare possibly because of synchrotron self-absorption.

Comparison of the outburst of 1994 with those of 1971 and 1983 shows that there is a clear correlation between power of outburst and spectral energy distribution of variable source in each event; the larger is the outburst, the bluer is the variable source, i.e. in more powerful outbursts the energy spectrum of relativistic electrons is flatter.

Acknowledgements. The authors are grateful to all participants of OJ-94 Project for collaboration. V.A.H.-Th. would like to thank for hospitality the Tuorla Observatory where this work was partly completed. LOT, AS and TP would like to thank Finnish Academy for support, V.A.H.-Th. and SGM are grateful for financial support to Russian Federal Program "Astronomy" and Russian Fund of the Basic Researches (grant 98-02-16609).

\section{References}

Choloniewski J., 1981, Acta Astron. 31, 293

Dultzin-Hacyan D., Takalo L.O., Benitez E., et al., 1997, Rev. Mex. AA 33, 17

Efimov Yu.S., Shakhovskoy N.M., 1996, Tuorla Obs. Reports, Inf. 176, 32

Hagen-Thorn V.A., 1987, Thesis, LGU

Hagen-Thorn V.A., 1997, Astron. Lett. 23, 19

Hagen-Thorn V.A., Gataullina A.I., Mikolaichuk O.V., 1991, Astrofizika 34, 212 (Engl. transl.)

Hagen-Thorn V.A., Marchenko S.G., Mikolaichuk O.V., 1992, in "Variability of Blazar", Valtaoja E. \& Valtonen M.J. (eds.). Cambridge Univ. Press, p. 85

Hagen-Thorn V.A., Yakovleva V.A., Takalo L.O., Sillanpää, 1994, A\&A 290, 1

Lehto H., Valtonen M., 1996, ApJ 460, 207

Kidger M., Gonzalez-Perez J.N., 1994, Tuorla Obs. Reports, Inf. 174, 49

Kidger M., et al., 1998 (in preparation)

Marscher A.P., 1997, Pubblicazioni Osservatorio Astronomico Universita di Perugia 3, 81

Mead A.R.G., Ballard K.R., Brand P.W.J.L., et al., 1990, A\&AS 83, 183

Pacholczyk A.G., 1977, Radio Galaxies. Pergamon Press, Oxford, Chapter 2

Pian E., Treves A., Webb J., et al., 1996, Tuorla Obs. Reports, Inf. 176,26

Pursimo T., Lehto H.J., 1996, Tuorla Obs. Reports, Inf. 176, 2

Sillanpää A., Haarala S., Valtonen M.J., et al., 1988, ApJ 325, 628

Sillanpää A., Takalo L.O., Pursimo T., et al., 1996a, A\&A 305, L17

Sillanpää A., Takalo L.O., Pursimo T., et al., 1996b, A\&A 315, L13

Smith P.S., Balonek T.J., Elston R., Heckert P.A., 1987, ApJS 64,459

Takalo L.O., 1994, Vistas Astron. 38, 77

Urry M., Padovani P., 1996, PASP 107, 80

Wald A., 1940, Ann. Math. Statistics 11, 284 